\title{
Fixed Point Theorems of Hegedus Contraction Mapping in Some Types of Distance Spaces
}

\author{
M. A. Ahmed ${ }^{1}$, F. M. Zeyada ${ }^{1}$, G. F. Hassan ${ }^{1,2}$ \\ ${ }^{1}$ Department of Mathematics, Faculty of Science, Assiut University, Assiut, Egypt \\ ${ }^{2}$ Department of Mathematics, Teachers' College, Arar, KSA \\ Email:mahmed68@yahoo.com, zeyada1@hotmail.com,gamal6@yahoo.com
}

Received May 22, 2012; revised June 12, 2012; accepted July 12, 2012

\begin{abstract}
In the present paper, we prove some fixed point theorems of Hegedus contraction in some types of distance spaces, dislocated metric space, left dislocated metric space, right dislocated metric space and dislocated quasi-metric metric space which are generalized metrics spaces where self-distances are not necessarily zero.
\end{abstract}

Keywords: Distance Space; Dislocated Metric Spaces; Quasi-Metric Spaces; Dislocated Quasi-Metric Spaces; Contraction

\section{Introduction}

Fixed point theorems on metric spaces and generalized types of metric spaces have applications in the area of logic programming semantics (see, e.g., [1-3]). Dislocated metrics are also known as metric domains in the context of domain theory [4]. The slightly less general notation of partial metrics was also studied in [5].

The definition of a distance space was introduced by $\mathrm{P}$. Waszkiewick [6,7].

Definition 1.1 [6,7]. Let $X$ be a set. A distance on $X$ is a map $d: X \times X \rightarrow[0, \infty)$. A pair $(X, d)$ is called a distance space.

Definition 1.2. Let $(X, d)$ be a distance space. Consider the following conditions:

Following Waszkiewick [4], let $X$ be a set. A distance on $X$ is a map $d: X \times X \rightarrow[0, \infty)$. A pair $(X, d)$ is called a distance space. If $d$ satisfies the following conditions:

$$
\begin{array}{ll}
\left(D M_{1}\right) & d(x, x)=0 \quad \forall x \in X \\
\left(D M_{2}\right) & d(x, y)=d(y, x)=0 \Rightarrow x=y \\
\left(D M_{3}\right) & d(x, y)=d(y, x) \\
\left(D M_{4}\right) & d(x, y) \leq d(x, z)+d(z, y) \\
\left(D M_{5}\right) & x=y \text { if } f d(x, x)=d(x, y)=d(y, y) \\
\left(D M_{6}\right) & d(x, x) \leq d(x, y) \\
\left(D M_{7}\right) & d(x, z) \leq d(x, y)+d(y, z)-d(y, y)
\end{array}
$$

for all $x, y, z \in X$. If $d$ satisfies $\left(D M_{1}\right)-\left(D M_{4}\right)$, then it is called a metric on $X$. If it satisfies conditions $\left(D M_{2}\right)-$
$\left(D M_{4}\right)$ it is called a dislocated metric [1,2] (or simply d-metric) on $X$. If it satisfies conditions $\left(D M_{1}\right),\left(D M_{2}\right)$, and $\left(D M_{4}\right)$, It is called a quasi-metric (or simply q-metric) on $X$ [8]. If it satisfies conditions $\left.\left(D M_{2}\right)\right)$ and $\left(D M_{4}\right)$, It is called a dislocated quasi-metric (or simply dq-metric) on $X[1,2]$. If it satisfies conditions $\left(D M_{3}\right)$, $\left(D M_{5}\right),\left(D M_{6}\right)$ and $\left(D M_{7}\right)$, It is called a partial metric on $X[5]$.

It is clear that any partial metric is a $d$-metric and any $d$-metric is $d q$-metric.

Hitzler and Seda gave an alternative proof of Matthews's Theorem [4] as follows.

Theorem 1.1 [1]. Let $(X, d)$ be a complete d-metric space and let $f: X \rightarrow X$ be a Banach contraction function. Then $f$ has a unique fixed point.

The plan of this paper is as follows. In Section 2, we introduce some definitions in distance spaces. In Section 3 , we establish some fixed point theorems in some types of distance space. In Section 4, we establish some fixed point theorems in some types of dislocated metric space.

\section{Some Basic Concepts and Results in Distance Spaces}

In the following we proceed with definitions which are needed for our results in a distance space. As it turns out, these notions can be carried over directly from conventional metrics.

Definition 2.1. A sequence $\left(x_{n}\right)$ in a distance space $(X$, $d)$ ld-converges (resp. $\boldsymbol{r d}$-converges, $d$-converges) to $x \in X$ if $\lim _{n \rightarrow \infty} d\left(x_{n}, x\right)=0$ (resp. $\lim _{n \rightarrow \infty} d\left(x_{n}, x\right)=0$, $\left.\lim _{n \rightarrow \infty} d\left(x_{n}, x\right)=\lim _{n \rightarrow \infty} d\left(x, x_{n}\right)=0\right)$. In this case, $x$ is 
called the Id-limit (resp. $\boldsymbol{r d}$-limit, $\boldsymbol{d}$-limit) of the sequence $\left(x_{n}\right)$.

Is is obvious that a $d$-limit of a sequence $\left(x_{n}\right)$ is $l d$ limit and $r d$-limit but the converse may not be true.

Definition 2.2. A sequence $\left(x_{n}\right)$ in a distance space $(X$, $d$ ) is called Cauchy if $\lim _{m, n \rightarrow \infty} d\left(x_{m}, x_{n}\right)=0$.

Definition 2.3. A distance space $(X, d)$ is called Idcomplete (resp. $\boldsymbol{r d}$-complete, $\boldsymbol{d}$-complete) if every Cauchy sequence in it is ld-convergent (resp. $r d$-convergent, $d$-convergent).

Definition 2.4. Let $\left(X, d_{1}\right)$ and $\left(Y, d_{2}\right)$ be distance spaces. Then $f: X \rightarrow Y$ is sequentially l-continuous if $\forall x_{0} \in X, \forall \varepsilon>0, \exists \delta(\varepsilon)>0$ such that

$$
d_{1}\left(x, x_{0}\right)<\delta(\varepsilon) \Rightarrow d_{2}\left(f(x), f\left(x_{0}\right)\right)<\varepsilon .
$$

Definition 2.5. Let $\left(X, d_{1}\right)$ and $\left(Y, d_{2}\right)$ be distance spaces. Then $f: X \rightarrow Y$ is sequentially r-continuous if $\forall x_{0} \in X, \forall \varepsilon>0, \exists \delta(\varepsilon)>0$ such that

$$
d_{1}\left(x_{0}, x\right)<\delta(\varepsilon) \Rightarrow d_{2}\left(f\left(x_{0}\right), f(x)\right)<\varepsilon .
$$

We state the following lemmas without proof.

Lemma 2.1. Let $\left(X, d_{1}\right)$ and $\left(Y, d_{2}\right)$ be distance spaces. A function $f: X \rightarrow Y$ is sequentially $l d$-continuous (resp. $r d$-continuous, $d$-continuous) if and only if for each sequence $\left(x_{n}\right)$ which is $l d$-convergent (resp. $r d$-convergent, $d$-convergent) to $x_{0} \in X$, the sequence $\left(f\left(x_{n}\right)\right)$ is $l d$ convergent (resp. $r d$-convergent, $d$-convergent) to $f\left(x_{0}\right) \in Y$.

Lemma 2.2. Let $\left(X, d_{1}\right)$ and $\left(Y, d_{2}\right)$ be distance spaces. Then $f: X \rightarrow Y$ is sequentially $d$-continuous if $f$ is sequentially $l d$-continuous and sequentially $r d$-continuous.

The following counterexample illustrates the reverse of Lemma 2.2 need not be true.

Counterexample 2.1. Let $d: N \times N \rightarrow[0, \infty)$ be defined by:

$$
d(x, 1)=\frac{1}{x} \quad \forall x \in N-\{1\},
$$

$\mathrm{d}(x, y)=2, \quad \forall x \in N, \quad y \in N-\{1\}, d(1,1)=2$.

Let $f: N \times N$ defined by $f(x)=x, \forall x \in N-\{1\}, f(1)=2$. Since $\left(x_{n}\right)\left(x_{n}=n \forall n \in N-\{1\}\right)$ converges to 1 but $\left(f\left(x_{n}\right)\right.$ does not converges to $f(1)$, then $f$ is not sequentially $l$-continuous. But $f$ is sequentially $d$-continuous.

Now, we give the following lemma without proof.

Lemma 2.4. Every subsequence of $l d$-convergent (resp. $r d$-convergent, $d$-convergent) sequence to $x_{0}$ is $l d$-convergent (resp. $r d$-convergent, $d$-convergent) to $x_{0}$.

It is obvious that the converse of Lemma 2.4 may not be true.

Definition 2.6. Let $(X, d)$ be a distance space. Let $N$ denote the set of positive integers and $N_{0}=N \cup\{0\}$. For any set $Y \subseteq X$ the diameter of the set $Y$ is defined by $\operatorname{diam} Y=\sup \{d(x, y: x, y \in Y\}$ and

$$
O(x)\left\{f^{k} x: k \in N_{0}\right\}
$$

is the $f$-orbit of $x$. The point $x \in X$ is called regular (f-regular) if $\operatorname{diam} O(x)<+\infty$.

A function $f:(X, d) \rightarrow(X, d)$ is called a Hegedus contraction [9] if there exists $0 \leq \lambda \leq 1$ such that

$$
\begin{aligned}
& d(f x, f y) \\
\leq & \lambda \operatorname{diam}\left\{x, y, f x, f y, f^{2} x, f^{2} y, \cdots\right\}, \\
= & \lambda \operatorname{diam}[O(x) \cup O(y)]
\end{aligned}
$$

where $\forall x \in X, x$ is $f$-regular.

Lemma 2.2. Let $(X, d)$ be a distance space.

If $f:(X, d) \rightarrow(X, d)$ is a Hegedus contraction function, then for any $n \in N$ and $x, y \in X$,

$$
d\left(f^{n} x, f^{n} y\right) \leq \lambda^{n}[O(x) \cup O(y)] .
$$

Proof. Since

$$
\forall f^{k} x, f^{h} y \in\left(O\left(f^{n-1} x\right) \cup O\left(f^{n-1} y\right)\right)
$$

where $k \geq n-1, h \geq n-1$,

$$
d\left(f^{k} x, f^{h} y\right) \leq \lambda \operatorname{diam}\left[O\left(f^{n-2} x\right) \cup O\left(f^{n-2} y\right)\right],
$$

then

$$
\begin{aligned}
d\left(f^{n}(x), f^{n}(y)\right) & \leq \lambda \operatorname{diam}\left[O\left(f^{n-1} x\right) \cup O\left(f^{n-1} y\right)\right] \\
& \leq \lambda^{2} \operatorname{diam}\left[O\left(f^{n-2} x\right) \cup O\left(f^{n-2} y\right)\right] \\
& \vdots \\
& \leq \lambda^{n} \operatorname{diam}[O(x) \cup O(y)] .
\end{aligned}
$$

\section{Fixed Point Theorems in Distance Spaces}

In this section, we introduce some fixed point theorems in distance space.

Definition 3.1. A distance space $(X, d)$ is called an $\boldsymbol{I d}$-Hausdorff (resp. $\boldsymbol{r d}$-Hausdorff, $\boldsymbol{d}$-Hausdorff) space iff the $l d$-limt (resp. $r d$-limt, $d$-limt) of $l d$-convergent (resp. $r d$-convergent, $d$-convergent) sequence is unique. Such that $\forall x \in X$, $\operatorname{diam}(O(x))<+\infty$.

Lemma 3.1. Let $(X, d)$ be a distance space such that $\forall x \in X, \operatorname{diam}(O(x))<+\infty$. If a function $f: X \rightarrow X$ is a Hegedus contraction, then $\left(f^{n}\left(x_{0}\right)\right.$ is a Cauchy sequence for each $x_{0} \in X$.

Proof. Choose any $x_{0} \in X$, for any integer $r \in N \cup\{0\}$. By lemma 2.2,

$$
\begin{aligned}
& d\left(f^{n}\left(x_{0}\right), f^{n+r}\left(x_{0}\right)\right) \\
\leq & \lambda^{n} \operatorname{diam}\left[O\left(x_{0}\right) \cup O\left(f^{r}\left(x_{0}\right)\right)\right] . \\
= & \lambda^{n} \operatorname{diam} O\left(x_{0}\right)
\end{aligned}
$$

The last term tends to zero as $n$ tends to infinity. Also, 
we proceed similarly as above and obtain

$$
\begin{aligned}
& d\left(f^{n+r}\left(x_{0}\right), f^{n}\left(x_{0}\right)\right) \\
\leq & \lambda^{n} \operatorname{diam}\left[O\left(f^{r}\left(x_{0}\right) \cup O\left(x_{0}\right)\right] .\right. \\
= & \lambda^{n} \operatorname{diam} O\left(x_{0}\right)
\end{aligned}
$$

The last term tends to zero as $n$ tends to infinity. Thus, $\left(f^{n}\left(x_{0}\right)\right.$ is a Cauchy sequence.

Theorem 3.1. Let $(X, d)$ be a $l d$-Hausdorff $l d$-complete distance space and a function $f: X \rightarrow X$ be a $l d$-continuous Hegedus contraction mapping such that $\forall x \in X, x$ is $f$-regular. Then $f$ has a fixed point.

Proof. From Lemma 3.1, $\left(f^{n}\left(x_{0}\right)\right.$ is a Cauchy sequence. Since $(X, d)$ is $l d$-complete distance space, then $\left(f^{n}\left(x_{0}\right)\right.$ is $l d$-convergent, say to $x \in X$. From the $l d$-continuity of the mapping $f,\left(f^{n+1}\left(x_{0}\right)\right) l d$-converges to $f(x)$. From Lemma 2.1, $\left(f^{n+1}\left(x_{0}\right) l d\right.$-converges to $x$. Since $(X, d)$ is ld -Haus- dorff, then $f(x)=x$.

In a similar manner of Theorem 3.1, we can prove the following theorems.

Theorem 3.2. Let $(X, d)$ be a $r d$-Hausdorff $r d$-complete distance space and a function $f: X \rightarrow X$ be a $r d$-continuous Hegedus contraction mapping such that $\forall x \in X$, $x$ is $f$-regular. Then $f$ has a fixed point.

Theorem 3.3. Let $(X, d)$ be a d-Hausdorff $d$-complete distance space and a function $f: X \rightarrow X$ be a $d$-continuous Hegedus contraction mapping such that $\forall x \in X, x$ is $f$-regular. Then $f$ has a fixed point.

\section{Fixed Point Theorems in Types of Dislocated Metric Spaces}

In this section, we introduce the concept of left dislocated metric spaces and right dislocated metric spaces. Also, we state and prove some fixed point theorems in these spaces. Furthermore, we prove fixed point theorem in dislocated quasi-metric spaces and in dislocated metric spaces.

Definition 4.1. A left dislocated metric (simply Id-metric) is a distance $d$ on a set $X$ satisfying the following axioms, for all $x, y, z \in X$,

$$
\begin{aligned}
& \left(L D_{1}\right) \text { if } d(x, y)=d(y, x)=0 \text { then } x=y ; \\
& \left(L D_{2}\right) d(x, y) \leq d(z, x)+d(z, y) .
\end{aligned}
$$

Lemma 4.1. $l d$-limits in $l d$-metric spaces are unique.

Proof. Let $x$ and $y$ be $l d$-limits of the sequence $\left(x_{n}\right)$. By property $\left(L D_{2}\right)$, it follows that

$$
d(x, y) \leq d\left(x_{n}, x\right)+d\left(x_{n}, y\right) \rightarrow 0 \text { as } n \rightarrow \infty .
$$

Hence, $d(x, y)=0$. In a similar way, one can deduce that $d(y, x)=0$. So, we obtain from Property $\left(L D_{1}\right)$ that $x=y$.

Theorem 4.1. Let $(X, d)$ be a $l d$-complete $l d$-metric space and a function $f: X \rightarrow X$ be $l d$-continuous Hegedus contraction function such that $\forall x \in X, x$ is $f$-regular. Then $f$ has a fixed point.

Proof. Existence. From Lema 3.1, $\left(f^{n}\left(x_{0}\right)\right)$ is a Cauchy sequence. Since $(X, d)$ is $l d$-complete $l d$-metric then $\left(f^{n}\left(x_{0}\right)\right) l d$-convergent, say to $x, f$ is $l d$-continuous and so from Lemmas 2.1 and 4.1,

$$
\begin{aligned}
f(x) & =f\left(\lim _{n \rightarrow \infty} f^{n}\left(x_{0}\right)\right) \\
& =\lim _{n \rightarrow \infty} f^{n+1}\left(x_{0}\right) . \\
& =x
\end{aligned}
$$

Uniqueness. Suppose that there are two fixed points $x$ and $y$. Then $f(x)=x, f(y)=y$ and

$$
\begin{aligned}
d(x, y) & =d(f(x),(y)) \\
& \leq \lambda \operatorname{diam}[O(x) \cup O(y)] \\
& =\lambda \operatorname{diam}\left\{x, y, f x, f y, f^{2} x, f^{2} y, f^{3} x, f^{3} y, \cdots\right\} \\
& =\operatorname{diam}\{x, y, x, y, x, y, x, y, \cdots\} \\
& =\lambda \max \{d(x, y), d(y, x), d(x, x), d(y, y)\} .
\end{aligned}
$$

If $\max \{d(x, y), d(y, x), d(x, x), d(y, y)\}$ is $d(x, y)$ or $d(y$, $x)$ or $d(x, x)$ or $d(y, y)$, then one can deduce that $d(x, y)=$ $d(y, x)=d(x, x)=d(y, y)=0$. Hence from $\left(L D_{1}\right), x=y$.

Definition 4.2. A right dislocated metric (simply rd-metric) is a distance $d$ on a set $X$ satisfying the following axioms, for all $x, y, z \in X$,

$$
\begin{aligned}
& \left(R D_{1}\right) \text { if } d(x, y)=d(y, x)=0 \text { then } x=y \\
& \left(R D_{2}\right) d(x, y) \leq d(x, z)+d(y, z) .
\end{aligned}
$$

In a similar proof of Lemmas 4.1, we give the following lemmas:

Lemma 4.2. $r d$-limits in $r d$-metric spaces are unique.

Lemma 4.3. $d$-limits in $d q$-metric spaces are unique.

Lemma 4.4. $d$-limits in $d$-metric spaces are unique.

In a similar manner of Theorem 4.1 , one can have the following theorems.

Theorem 4.2. Let $(X, d)$ be a $r d$-complete $r d$-metric space and a function $f: X \rightarrow X$ be rd-continuous Hegedus contraction function such that $\forall x \in X, x$ is $f$-regular. Then $f$ has a fixed point.

Theorem 4.3. Let $(X, d)$ be a $d$-complete $d q$-metric space and a function $f: X \rightarrow X$ be $d$-continuous Hegedus contraction function such that $\forall x \in X, x$ is $f$-regular. Then $f$ has a fixed point.

Theorem 4.4. Let $(X, d)$ be a $d$-complete $d$-metric space and a function $f: X \rightarrow X$ be d-continuous Hegedus contraction function such that $\forall x \in X, x$ is $f$-regular. Then $f$ has a fixed point.

\section{REFERENCES}

[1] P. Hitzler, "Generalized Metrics Topology in Logic Pro- 
gramming Semantics," Ph.D. Thesis, National University of Ireland (University College Cork), Dublin, 2001.

[2] P. Hitzler and A. K. Seda, "Dislocated Topologies," Journal of Electrical Engineering, Vol. 51, No. 12/s, 2000, pp. 3-7.

[3] A. K. Seda, "Quasi Metrics and the Semantics of Logic Programs," Fundamenta Informaticae, Vol. 29, No. 1, 1997, pp. 97-117.

[4] S. G. Matthews, "Metric Domains for Completeness," Ph.D. Thesis, University of Warwick, Coventry, 1986.

[5] S. G. Matthews, "The Topology of Partial Metric Spaces," Research Repodt 22, University of Warwick, Coventry, 1992, 19 p.
[6] P. Waszkiewicz, "The Local Triangle Axioms in Topology and Domain Theory," Applied General Topology, Vol. 4, No. 1, 2003, pp. 47-70.

[7] P. Waszkiewicz, "Quantitative Continuous Domains," Ph.D. Thesis, The Universiyy of Birminghham, Edgbaston, 2002.

[8] J. J. M. M. Rutten, "Elements of Generalized Ultrametric Domain Theory," Theoretical Computer Science, Vol. 170, No. 1-2, 1996, pp. 349-381. doi:10.1016/S0304-3975(96)80711-0

[9] M. Hegedus, "Some Extension of Fixed Point Theorems," Publications de l'Institut Mathématique (Beograd), Vol. 27. No. 41, 1980, pp. 77-82. 